\title{
Editorial Letter: The Road Ahead
}

\section{Charles-Étienne Benoit, Piotr Kałowski, and Konrad Janowski}

University of Economics and Human Sciences in Warsaw

\section{DEAR READERS, AUTHORS, REVIEWERS, AND EDITORIAL BOARD MEMBERS OF ADVANCES IN COGNITIVE PSYCHOLOGY}

It has been more than two years since our previous newsletter. Much has happened since and it is important to look back and write to you all about the major changes that occurred under the supervision of Dr. Konrad Janowski, the Editor-in-Chief of Advances in Cognitive Psychology (ACP).

First, it is important to highlight that the journal's publisher has been updated. At its core, the structure supporting the journal remains the same. However, our university, previously known as the University of Finance and Management has moved to a brand-new campus and rebranded as the University of Economics and Human Sciences in Warsaw. Accordingly, the University now carries the label of our journal's publisher for international recognition. We at ACP are grateful to the financial director of the educational group Vizja, Mr. Krzysztof Godlewski, for the continuous support.

With this transition, Dr. Janowski was recently appointed as the university's Rector. To strengthen the ranks in ACP, Dr. CharlesEtienne Benoit was promoted as Co-Editor-in-Chief. Dr. Benoit was recruited at the University in late 2018 as an associate professor and is currently in charge of the journal's daily activities. His expertise as a neuroscientist centers around memory, rhythm, and motor rehabilitation.

Considering this recent transfer of leadership at ACP, these changes created certain voids that were in need to be filled, most notably in our abilities to index and showcase our publications in international scientific databases. Under the leadership of Dr. Janowski and Dr. Benoit, the journal was awarded funding by the Polish Ministry of Science and Higher Education. This was put to good use to build a platform facilitating the successful inclusion of articles in the National Center for Biotechnology (PubMed) Information search database. With this tool at our disposal, our production manager Piotr Kałowski and the newly recruited technical editors (Magdalena Telega and Valeryia Bareika) worked hard to bring our backlog up to date. Thus, in addition to other top-level databases such as Scopus or the Web of Science Group, we can now boast being up-to-date and available for free on PubMed.
Another achievement was the expansion of our Editorial Board. Several members of the old guard continue to support ACP. Accordingly, we wish to extend our gratitude to Dr. Lisi Beyersmann in Sydney (Australia), Dr. Claus-Christian Carbon in Bamberg (Germany), Dr. Hanna Chainay in Lyon (France), Dr. Simone Dalla Bella in Montreal (Canada), Dr. Kesong Hu in Sault Sainte Marie (USA), Dr. Markus Kiefer in Ulm (Germany), Dr. Ingrid Scharlau in Paderborn (Germany), Dr. Elisabet Service in Hamilton (Canada) and Dr. John Tivendel in Moncton (Canada). Furthermore, we invited several more scientists and today we welcome to the team Dr. Valentin Begel in Montreal (Canada), Dr. Mario Bogdanov in Montreal (Canada), Dr. Matthieu Boisgontier in Ottawa (Canada), Dr. Boris Cheval in Geneva (Switzerland), Dr. Cyril Forestier in LeMans (France), Dr. Patricia Garrido Vasquez in Concepción (Chili), Dr. Jacek Gierus in Warsaw (Poland), Dr. Juan Jose Ortells in Almería (Spain), Dr. Giuseppe Leonardi in Warsaw (Poland), Dr. Akito Miura in Tokyo (Japan), Dr. Floris van Vugt in Montreal (Canada) and Dr. Artyom Zinchenko in München (Germany). Our current team thus represents Europe, North and South America, Asia and Oceania. It is thanks to them all that the journal remains healthy, active, and able to diversify its scope without compromising its quality.

Looking back at some numbers from the past 2 years, we successfully published 28 articles in 2019 compared to 32 in 2020 . The amount of original research articles submitted to ACP went from 94 in 2019 to 145 in 2020 . Our acceptance rate went from $37 \%$ in 2019 to $24 \%$, demonstrating our desire to carefully select quality science to publish. toblish. Accordingly, with this new year, we are implementing some bold changes to nurture our brand and improve our status within the scientific community.

For our image, we decided to introduce a new, modern logo, made by professional in-house designers at our University, as well as to revitalize our online page with a more accessible and sleeker interface. This comes alongside a range of technical improvements to increase the website speed and security with a SSL protocol. For our ability to fur- 
ther reach and engage with the scientific community, we inaugurated a new twitter platform (@AdvCognPsychol) where we aim to distribute our articles as they are released. This comes with a change in our distribution policies that aim to promote the articles we publish as soon as they are ready while keeping a fixed quarterly volume numeration.

We have simultaneously revised and updated our editorial policies. We decided to widen the scope of our journal to include high-quality science from all areas and aspects of human cognitive psychology, including, but not limited to, perception, attention, memory, social cognition, and language processing in behavioral, cognitive, psychophysiological, and neuropsychological perspectives, as well as in computer- and modeling-based science. We will welcome original empirical and theoretical articles, as well as replications, reports of null findings, literature reviews and proposals for special issues.

ACP always was an Open Access journal, as advertised on journal databases such as DOAJ or Sherpa Romeo, but one of the biggest changes to be expected this year is the transition of our journal towards greater Open Science practices: We are aiming to significantly enhance publication transparency and replicability by adopting and promoting the Guidelines for Transparency and Openness Promotion (TOP). We will also welcome preprints and free-format submissions.

With all these new directions, we hope to honor the legacy of the journal founder, Dr. Piotr Jaśkowski, and keep ACP as an international caliber scientific journal.

We are thankful for your support and readership, we hope our journal inspires your scientific endeavors, and we are always looking forward to your manuscripts.

Sincerely yours,

Charles-Étienne Benoit, Co-editor-in-Chief

Piotr Kałowski, Production Manager

Konrad Janowski, Editor-in-Chief 